Pacific Journal of Mathematics

THE DUAL OF THE NILRADICAL OF THE PARABOLIC 


\title{
THE DUAL OF THE NILRADICAL OF THE PARABOLIC SUBGROUPS OF SYMPLECTIC GROUPS
}

\author{
ThOMAS A. FARMER
}

\begin{abstract}
For an arbitrary parabolic subgroup $P$ of the real or complex symplectic group, let $N$ be the nilradical. Using Kirillov theory, a subset of the dual of $N$ is found, whose complement has Plancherel measure zero. It is shown how these representations extend by combining with the oscillator representation of a lower rank symplectic group. A result is obtained concerning the commuting algebra of the restrictions to $P$ of the principal series representation of the symplectic group induced from a unitary character of the opposite parabolic.
\end{abstract}

Introduction. In [6] and [2] there are irreducibility theorems for principal series representations of symplectic groups induced from unitary characters of certain maximal parabolic subgroups. Such a representation can be realized to act in the $L^{2}$-space of a nilpotent subgroup, the nilradical of the opposite parabolic. The irreducibility results are obtained in two stages. In the first stage the representation $T$ is restricted to the opposite parabolic and the commuting algebra of the restriction is computed using nilpotent harmonic analysis. Since these parabolics are maximal subgroups, the full symplectic group is generated by the opposite parabolic together with a single element, say $p$. The commuting algebra of $T$ is, therefore, the subalgebra of the commuting algebra of the restriction consisting of operators that commute with $T(p)$. The second stage is the difficult determination of which operators these are. For arbitrary parabolic subgroups of the symplectic groups, even for arbitrary maximal ones, it appears that the second stage of this program is not feasible and the irreducibility theorems must come from more powerful methods in semisimple representation theory. However, the first stage can be carried out in full generality, and it is of interest for the way in which the oscillator representation occurs and because of the computations involved in the nilpotent harmonic analysis. This is the topic of this paper.

To be more specific, let $P$ be a parabolic subgroup of the symplectic group $\operatorname{Sp}(n, F)$, where $F=\mathbf{R}$ or $\mathbf{C}$. It is known that the principal series representation $T$ of $\operatorname{Sp}(n, F)$ induced up from a unitary character of $P$ can be realized to act in $L^{2}(N)$, where $N$ is a nilpotent subgroup of $\operatorname{Sp}(n, F)$ and $N \cap P$ is trivial. Let $M$ be the normalizer of $N$ in $P$, then the semidirect product $N M$ is a parabolic subgroup conjugate to $P$ ( $N M$ is 
the opposite parabolic) and $N$ is the nilradical of $N M$. These groups are further described in $\S 1$. In $\S 2$, the theory of Kirillov is used to compute, except for a set of measure zero, the dual object of the nilradical of an arbitrary parabolic subgroup of $\operatorname{Sp}(n, F)$. Denote by $\Re$ the Lie algebra of the nilradical $N$ and let $\mathfrak{T}^{*}$ be the real vector space dual of $\Re$. Sufficiently many coadjoint orbits in $\Re^{*}$ are found so that the complement of their union has measure zero in $\Re^{*}$. The irreducible representations corresponding to these orbits are computed and they form a subset of the dual object of $N$ whose complement must have Plancherel measure zero.

Let $F=\mathbf{C}$, to simplify the remainder of this introduction, and let $\lambda$ be an element of the dual of $N$, acting in a space $L^{2}(V)$. Define $M_{\lambda}$ to be the subgroup of $M$ consisting of all $m \in M$ such that the representations $z \rightarrow \lambda\left(\mathrm{m}^{-1} \mathrm{zm}\right)$ and $\lambda$ are unitarily equivalent. Depending on the choice of $P$, we will see that $M$ is isomorphic to a direct product of the form

$$
\operatorname{Sp}\left(n_{0}, \mathrm{C}\right) \times \mathrm{Gl}\left(n_{1}, \mathrm{C}\right) \times \cdots \times \mathrm{Gl}\left(n_{r}, \mathrm{C}\right) .
$$

where $n=n_{0}+n_{1}+\cdots+n_{r}, n_{0}$ is nonnegative and $n_{1}, \ldots, n_{r}$ are positive. It turns out that $M_{\lambda}$ is isomorphic to

$$
\operatorname{Sp}\left(n_{0}, \mathbf{C}\right) \times O\left(n_{1}, \mathbf{C}\right) \times \cdots \times O\left(n_{r}, \mathbf{C}\right) .
$$

In $\S 3$, we extend $\lambda$ to a unitary representation of $N M_{\lambda}$ acting in the same Hilbert space $L^{2}(V)$. For this we need a representation $D_{\lambda}$ of $M_{\lambda}$ acting in $L^{2}(V)$ and satisfying

$$
\lambda\left(m^{-1} z m\right)=D_{\lambda}(m)^{-1} \lambda(z) D_{\lambda}(m) \quad\left(z \in N, m \in M_{\lambda}\right) .
$$

The operators $D_{\lambda}(m)$ are given in terms of the oscillator representation of $\operatorname{Sp}\left(n_{0}, \mathbf{C}\right)$ and a certain natural representation of $O\left(n_{1}, \mathbf{C}\right)$ $\times \cdots \times O\left(n_{r}, \mathrm{C}\right)$.

Finally, in $\S 4$, we indicate that there is a *-isomorphism between the commuting algebras of $\left.T\right|_{N M}$ and $D_{\lambda}$. Throughout the paper we give the analogous results for the real field, which are more complicated to state.

1. Definitions. In this section, we define some of the groups and introduce some of the notation that will be used throughout the paper.

Let $F$ be the real field or the complex field, $\mathbf{R}$ or $C$. We choose to define the symplectic groups over $F$ as is done in [5] in order to have the most appealing treatment of the parabolic subgroups. Because of this choice, a notation for "secondary transpose" becomes very useful. For any positive integer $p$, let

$$
J_{p}=\left[\begin{array}{lll} 
& & \\
& \cdot & \\
1 & &
\end{array}\right]
$$


be the $p \times p$ matrix with ones along the secondary diagonal and zeros elsewhere. The subscript can be omitted when the context determines the dimensions of $J_{p}$. If $x$ is a $p \times q$ matrix and $x^{t}$ denotes the usual transpose of $x$, let the secondary transpose $x^{s}$ be defined by

$$
x^{s}=J_{q} X^{t} J_{p}
$$

It is easily verified that $\left(x^{s}\right)^{t}=\left(x^{t}\right)^{s}$ and, in the case of invertible matrices, $\left(x^{s}\right)^{-1}=\left(x^{-1}\right)^{s}$. Thus, we can write $x^{s t-1}$ for $\left(\left(x^{s}\right)^{t}\right)^{-1}$ without confusion. Also, $(x y)^{s}=y^{s} x^{s}$ whenever $x$ and $y$ are compatible for multiplication.

Fix a positive integer $n$ and define the symplectic group

$$
\operatorname{Sp}(n, F)=\left\{g \in F^{2 n \times 2 n}: g\left[\begin{array}{ll}
0 & -J_{n} \\
J_{n} & 0
\end{array}\right] g^{t}=\left[\begin{array}{ll}
0 & -J_{n} \\
J_{n} & 0
\end{array}\right]\right\} .
$$

Another way of expressing (1.1) is the following: denote the elements of $F^{1 \times 2 n}$ by $[x y]$ with $x, y \in F^{1 \times n}$. Then $\operatorname{Sp}(n, F)$ is the subgroup of $\mathrm{Gl}(2 n, F)$ consisting of all linear transformations of $F^{1 \times 2 n}$ which leave invariant the bilinear form

$$
B\left(\left[x_{1} y_{1}\right],\left[x_{2} y_{2}\right]\right)=y_{1} x_{2}^{s}-x_{1} y_{2}^{s} .
$$

The parabolic subgroups of $\operatorname{Sp}(n, F)$, up to conjugacy, can be worked out from (1.2) as in [8] (\$8). Let

$$
\left\{n_{1}, n_{2}, \ldots, n_{r}, 2 n_{0}, n_{r}, n_{r-1}, \ldots, n_{1}\right\}
$$

be any sequence of integers with the indicated symmetry and satisfying the conditions that $n_{i}$ is positive for $i=1, \ldots, r, n_{0}$ is nonnegative, and $n=n_{0}+n_{1}+\cdots+n_{r}$. Consider the blocking scheme for $2 n \times 2 n$ matrices in which the diagonal blocks have dimensions

$$
n_{1} \times n_{1}, n_{2} \times n_{2}, \ldots, n_{r} \times n_{r}, 2 n_{0} \times 2 n_{0}, n_{r} \times n_{r}, \ldots, n_{1} \times n_{1},
$$

respectively, from upper left to lower right. Corresponding to this blocking scheme we have

$$
P=\{g \in \operatorname{Sp}(n, F): g \text { is upper block-triangular }\} .
$$

That is, $g \in P$ if and only if $g \in \operatorname{Sp}(n, F)$ and, with respect to the blocking scheme, the blocks in $g$ below the block-diagonal are zero. As defined by (1.3), $P$ is a parabolic subgroup of $\operatorname{Sp}(n, F)$. Moreover, each conjugacy class of parabolics in $\operatorname{Sp}(n, F)$ contains exactly one member of this form.

Also associated with the above blocking scheme are the subgroups of $\operatorname{Sp}(n, F)$ called $N$ and $M$, which we now describe. First, denote by $W$ the subgroup of $\operatorname{Gl}\left(n-n_{0}, F\right)$ consisting of all matrices that are lower 
block-triangular with identity blocks along the block-diagonal; here the blocking scheme for $\left(n-n_{0}\right) \times\left(n-n_{0}\right)$ matrices has diagonal blocks of dimensions

$$
n_{r} \times n_{r}, \ldots, n_{2} \times n_{2}, n_{1} \times n_{1},
$$

respectively, from upper left to lower right. Define $N$ to be the subgroup consisting of all lower block-triangular elements of $\operatorname{Sp}(n, F)$ with identity blocks along the diagonal. Using (1.1), a matrix computation shows that

$$
\begin{aligned}
& \text { (1.4) } N=\left\{\begin{array}{llll}
w^{s-1} & 0 & 0 & 0 \\
-x^{s} w^{s-1} & I & 0 & 0 \\
y^{s} w^{s-1} & 0 & I & 0 \\
t & y & x & w
\end{array}\right]: w \in W ; x, y \in F^{\left(n-n_{0}\right) \times n_{0}} \\
& \left.w t^{s}-t w^{s}=y x^{s}-x y^{s}\right\}
\end{aligned}
$$

We will abbreviate the notation for elements of $N$ by using $(t, y, x, w)$. The multiplication law for $N$ can then be written as

$$
\begin{aligned}
& \left(t_{1}, y_{1}, x_{1}, w_{1}\right)\left(t_{2}, y_{2}, x_{2}, w_{2}\right) \\
& =\left(\left(t_{1}-y_{1} x_{2}^{s}+x_{1} y_{2}^{s}+w_{1} t_{2} w_{2}^{s}\right) w_{2}^{s-1}, y_{1}+w_{1} y_{2}, x_{1}+w_{1} x_{2}, w_{1} w_{2}\right) .
\end{aligned}
$$

Let $M$ be the block-diagonal subgroup of $\operatorname{Sp}(n, F)$. If we denote by $\mathrm{Gl}\left(n_{r}, F\right) \times \cdots \times \mathrm{Gl}\left(n_{1}, F\right)$ the subgroup of $\mathrm{Gl}\left(n-n_{0}, F\right)$ consisting of block-diagonal matrices

$$
a=\left[\begin{array}{lll}
a_{r} & & \\
& \ddots & \\
& & a_{1}
\end{array}\right],
$$

with $a_{i} \in \mathrm{Gl}\left(n_{i}, F\right)$ for $i=1, \ldots, r$, then

(1.6) $M=\left\{\left[\begin{array}{ccc}a^{s-1} & 0 & 0 \\ 0 & s & 0 \\ 0 & 0 & a\end{array}\right]: s \in \operatorname{Sp}\left(n_{0}, F\right)\right.$,
$\left.a \in \operatorname{Gl}\left(n_{r}, F\right) \times \cdots \times \operatorname{Gl}\left(n_{1}, F\right)\right\}$.

In (1.4), (1.6), and throughout the paper, we use the convention that any matrix with $n_{0}$ as a dimension is suppressed if $n_{0}=0$. 
It is easy to verify that $N M$ is a semidirect product with $M$ normalizing $N$ and that $N M$ is conjugate to $P$ in $\operatorname{Sp}(n, F)$. Let $\chi$ be a unitary character of $P$. The unitary representation of $\operatorname{Sp}(n, F)$ induced by $\chi$ is a member of the principal series associated with $P$. The formula for this representation $T$ realized to act in $L^{2}(N)$ can be found in [5] (\$33.1). We shall only present, here, the formula for the restriction to $N M$ of the principal series representations of $\operatorname{Sp}(n, F)$. For $f \in L^{2}(N), z \in N$, and $m \in M$,

$$
T(z m) f(\zeta)=\delta(m)^{-1 / 2} \chi(m) f\left(m^{-1} \zeta z m\right),
$$

where $\delta(m)=d\left(m \zeta m^{-1}\right) / d \zeta$ and $d \zeta$ is Haar measure on $N$.

2. The dual of $\mathbf{N}$. Let the nilpotent group $N$ have the form (1.4). In particular, this means we are fixing a blocking scheme for $2 n \times 2 n$ matrices, in which the diagonal blocks have dimensions

$$
n_{1} \times n_{1}, \ldots, n_{r} \times n_{r}, 2 n_{0} \times 2 n_{0}, n_{r} \times n_{r}, \ldots, n_{1} \times n_{1},
$$

respectively, from upper left to lower right. This induces blocking schemes for the various submatrices of $z \in N$. Notice that if $(t, y, x, w) \in N$ then the blocking scheme induced on $t$ has blocks along the secondary diagonal of dimensions $n_{1} \times n_{1}, \ldots, n_{r} \times n_{r}$ from lower left to upper right.

In this section we use the method of Kirillov to obtain the dual object of $N$ (up to a set of Plancherel measure zero). For this we need the Lie algebra $\Re$, its real vector space dual $\Re^{*}$, and the coadjoint action of $N$ on $\mathfrak{T}^{*}$. The dual of $N$ is taken to be a set of irreducible unitary representations of $N$ which correspond to the orbits of maximal dimension in $\Re^{*}$ under the coadjoint action of $N$.

As a linear Lie algebra, $\Re$ is given by

(2.1) $\Re=\left\{\left[\begin{array}{llll}-w_{1}^{s} & 0 & 0 & 0 \\ -x_{1}^{s} & 0 & 0 & 0 \\ y_{1}^{s} & 0 & 0 & 0 \\ t_{1} & y_{1} & x_{1} & w_{1}\end{array}\right]: w_{1} \in \mathscr{W} ;\right.$

$$
\left.x_{1}, y_{1} \in F^{\left(n-n_{0}\right) \times n_{0}} ; t_{1}=t_{1}^{s}\right\} .
$$

where $W$, the Lie algebra of $W$, is the set of $\left(n-n_{0}\right) \times\left(n-n_{0}\right)$ lower block-triangular matrices with zero blocks along the diagonal. The best way to express $\Re^{*}$ seems to be as a set of equivalence classes in $F^{2 n \times 2 n}$. 
For $X, Y \in F^{2 n \times 2 n}$, define

$$
\langle X \mid Y\rangle=\operatorname{Re} \operatorname{tr} X Y^{t}
$$

(the real part of the trace of $X Y^{t}$ ). Let two elements, $X^{\prime}$ and $Y^{\prime}$, of $F^{2 n \times 2 n}$ be equivalent if $\left\langle X \mid X^{\prime}\right\rangle=\left\langle X \mid Y^{\prime}\right\rangle$ for all $X \in \mathcal{K}$. Denote the equivalence class of $X^{\prime}$ by $c\left(X^{\prime}\right)$, then

$$
\Re^{*}=\left\{c\left(\left[\begin{array}{llll}
-w_{1}^{s} & 0 & 0 & 0 \\
-x_{1}^{s} & 0 & 0 & 0 \\
y_{1}^{s} & 0 & 0 & 0 \\
t_{1} & y_{1} & x_{1} & w_{1}
\end{array}\right]\right): w_{1} \in \mathscr{W}, t_{1}=t_{1}^{s}\right\} .
$$

The adjoint (left) action of $N$ on $\mathscr{T}$ is given by $\operatorname{Ad} z(X)=z X z^{-1}$, for $z \in N$ and $X \in \Re$. Thus, the coadjoint (right) action of $N$ on $\Re^{*}$ is

$$
\operatorname{Ad}^{*} z\left(c\left(X^{\prime}\right)\right)=c\left(z^{t} X^{\prime} z^{t-1}\right) .
$$

This follows since, for $X \in \mathfrak{N}$, we have

$$
\begin{aligned}
\left\langle X \mid \operatorname{Ad}^{*} z\left(c\left(X^{\prime}\right)\right)\right\rangle & =\left\langle\operatorname{Ad} z(X) \mid c\left(X^{\prime}\right)\right\rangle \\
=\left\langle z X z^{-1} \mid X^{\prime}\right\rangle & =\left\langle X \mid z^{t} X^{\prime} z^{t-1}\right\rangle=\left\langle X \mid c\left(z^{t} X^{\prime} z^{t-1}\right)\right\rangle,
\end{aligned}
$$

using properties of the trace. If $X^{\prime}$ is lower block-triangular then $z^{t} X^{\prime} z^{t-1}$ is generally not lower block-triangular and this explains the need for equivalence classes.

For brevity, denote the elements of $\Re^{*}$ by $\left(t_{1}, y_{1}, x_{1}, w_{1}\right)$, keeping in mind that $\left(t_{1}, y_{1}, x_{1}, w_{1}\right)=\left(t_{2}, y_{2}, x_{2}, w_{2}\right)$ if and only if $t_{1}=t_{2}, y_{1}=y_{2}$, $x_{1}=x_{2}$, and $w_{1}$ is identical with $w_{2}$ below the block-diagonal. By computing the matrix product $z^{t} X^{\prime} z^{t-1}$, with $z=(t, y, x, w) \in N$ and $X^{\prime}=$ $\left(t_{1}, y_{1}, x_{1}, w_{1}\right) \in \mathcal{T}^{*}$, we find the formula

$$
\begin{aligned}
\operatorname{Ad}^{*} z\left(X^{\prime}\right)= & \left(w^{t} t_{1} w^{s t}, w^{t}\left(y_{1}+t_{1} x^{s t}\right),\right. \\
& \left.w^{t}\left(x_{1}-t_{1} y^{s t}\right), w^{t}\left(w_{1}-x_{1} x^{t}-y_{1} y^{t}-t_{1} t^{s t} w^{t}\right) w^{t-1}\right) .
\end{aligned}
$$

Letting $z$ vary in $N,(2.4)$ gives the orbit of $X^{\prime}$ in $\Re^{*}$ under the coadjoint action of $N$. In general, the orbit is very complicated but a considerable simplification occurs if $t_{1}$ has the property that each submatrix $t_{1}^{[k]}$, with $n_{1}+\cdots+n_{k}$ rows and columns, in the lower left corner of $t_{1}(k=1, \ldots, r)$ is non-singular, Let us call this property secondary block-diagonalizable (SBD) as suggested by the following matrix fact. Let $\Lambda$ be the set of all $\left(n-n_{0}\right) \times\left(n-n_{0}\right)$ matrices

$$
\lambda=\left[\begin{array}{lll} 
& & \lambda_{r} \\
& \cdot & \\
\lambda_{1} & &
\end{array}\right]
$$


with $\lambda_{i}=\lambda_{i}^{s} \in \operatorname{Gl}\left(n_{i}, F\right)(i=1, \ldots, r)$ along the second block-diagonal and zero elsewhere.

LEMMA 2.1. With respect to the blocking schemes indicated earlier, the matrix $t_{1}=t_{1}^{s}$ is $S B D$ if and only if $t_{1}=w^{t} \lambda w^{s t}$ for some $\lambda \in \Lambda$ and $w \in W$. Moreover, $\lambda$ and $w$ are unique in this case.

Proof. A statement proved in [5] (p. 59) can be applied to $t_{1} J=\left(t_{1} J\right)^{t}$ to yield this lemma.

The next result gives the maximal orbits in $\Re^{*}$ under the coadjoint action of $N$.

THEOREM 2.2. For $i=1,2$ let $\left(t_{i}, y_{i}, x_{i}, w_{i}\right) \in \mathfrak{T}^{*}$ and suppose $t_{1}$ is $S B D, t_{1}=w^{t} \lambda_{1} w^{s t}, w \in W, \lambda_{1} \in \Lambda$. Then (a) $\left(t_{1}, y_{1}, x_{1}, w_{1}\right)$ is in the same orbit as $\left(\lambda_{1}, 0,0,0\right)$, and (b) $\left(t_{1}, y_{1}, x_{1}, w_{1}\right)$ is in the same orbit as $\left(t_{2}, y_{2}, x_{2}, w_{2}\right)$ if and only if $t_{2}=v^{t} \lambda_{1} v^{s t}$ for some $v \in W$.

Proof. First we show that $\left(t_{1}, y_{1}, x_{1}, w_{1}\right)$ is in the same orbit as $\left(t_{1}, 0,0,0\right)$ by constructing a suitable $z \in N$. Recall that $z=(t, y, x, w) \in$ $N$ provided $w t^{s}-t w^{s}=y x^{s}-x y^{s}$. It follows that $\left(T-y x^{s}, y, x, I\right) \in N$ for any $T=T^{s}$. According to (2.4), to have

$$
\operatorname{Ad}^{*}\left(T-y x^{s}, y, x, I\right)\left(t_{1}, y_{1}, x_{1}, w_{1}\right)=\left(t_{1}, 0,0,0\right),
$$

we must set $y_{1}+t_{1} x^{s t}=0, x_{1}-t_{1} y^{s t}=0$ and find $T=T^{s}$ so that

$$
w_{1}-x_{1} x^{t}-y_{1} y^{t}-t_{1}\left(T-y x^{s}\right)^{s t} \cong 0 \text {. }
$$

where $\cong$ means these two matrices agree below the block-diagonal. Thus, let $x=t_{1}^{t-1} y_{1}^{s t}$ and $y=-t_{1}^{t-1} x_{1}^{s t}$ (since $t_{1}$ is SBD, it is invertible) and note that (2.5) is equivalent to

$$
t_{1} T^{t} \cong w_{1}+y_{1} x_{1}^{s} t_{1}^{-1} .
$$

We claim that $t_{1} A \cong B$ always has a solution $A=A^{s}$ when $t_{1}$ is SBD. The proof is a recursive construction of the block-columns of $A$. Here, $A$ and $B$ have a blocking scheme with diagonal blocks of dimensions $n_{1} \times n_{1}, \ldots, n_{r}$ $\times n_{r}$ from upper left to lower right. Let $A^{(j)}$ denote the $j$ th block-column of $A$. We will write $A^{(j)} \cong B^{(j)}$ when these block-column submatrices of $A$ and $B$ agree below the block-diagonal position. To begin, let

$$
X_{1}=\left[\begin{array}{c|c}
Y & 0 \\
\hline 0_{n_{r} \times n_{1}} & 0
\end{array}\right]
$$

be a solution to $t_{1} X_{1}^{(1)} \cong B^{(1)}$. Such a solution exists since $t_{1}$ is SBD and hence $t_{1}^{[r-1]}$ is invertible. Define $A_{1}=X_{1}+X_{1}^{s}$ then $A_{1}=A_{1}^{s}$ and $t_{1} A_{1}^{(1)}=$ $t_{1} X_{1}^{(1)} \cong B^{(1)}$. Suppose for some $k(1 \leq k<r-1)$ we have $A_{k}=A_{k}^{s}$ and 
$t_{1} A_{k}^{(j)} \cong B^{(j)}(j=1, \ldots, k)$. Let

$$
X_{k+1}=\left[\begin{array}{c|c|c}
0 & Y & 0 \\
\hline 0 & 0 & 0 \\
0 & 0 & 0
\end{array}\right] .
$$

Where $X_{k+1}^{(k+1)}=\left[\begin{array}{l}Y \\ 0\end{array}\right]$ and $Y$ is the $\left(n_{1}+n_{2}+\cdots+n_{r-k}\right) \times n_{k+1}$ submatrix of $X_{k+1}^{(k+1)}$ above the secondary block-diagonal. Since $t_{1}^{[r-k]}$ is invertible, there is such a matrix $X_{k+1}$ satisfying $t_{1} X^{(k+1)} \cong B^{(k+1)}-t_{1} A_{k}^{(k+1)}$. Let

$$
A_{k+1}=A_{k}+X_{k+1}+X_{k+1}^{s}
$$

then

$$
A_{k+1}=A_{k+1}^{s}, \quad t_{1} A_{k+1}^{(k+1)} \cong t_{1} A_{k}^{(k+1)}+t_{1} X_{k+1}^{(k+1)} \cong B^{(k+1)},
$$

and, for $1 \leq j \leq k$,

$$
t_{1} A_{k+1}^{(j)} \cong t_{1} A_{k}^{(j)} \cong B^{(j)} .
$$

The matrix $A=A_{r-1}$ is the desired solution. We have proved that (2.6) has a solution $T$ with $T=T^{s}$ and it follows that $\left(t_{1}, y_{1}, x_{1}, w_{1}\right)$ is in the same orbit as $\left(t_{1}, 0,0,0\right)$. Clearly, $(0,0,0, w) \in N$ and

$$
\operatorname{Ad}^{*}(0,0,0, w)\left(t_{1}, 0,0,0\right)=\left(\lambda_{1}, 0,0,0\right)
$$

by (2.4) so $\left(t_{1}, 0,0,0\right)$ is in the same orbit as $\left(\lambda_{1}, 0,0,0\right)$ and (a) is proved. The proof of (b) is straightforward.

If $t_{1}$ is SBD then we say that the orbit of $\left(t_{1}, y_{1}, x_{1}, w_{1}\right) \in \Re^{*}$ is an SBD orbit. By Theorem 2.2 the SBD orbits are in $1: 1$ correspondence with the set $\Lambda$. Next, we will show that SBD orbits are orbits of maximal dimension. Let $m$ denote the number of entries below the block diagonal in a blocking scheme corresponding to $n-n_{0}=n_{1}+\cdots+n_{r}$ so that $\operatorname{dim} W=m$.

THEOREM 2.3. (a) Each SBD orbit is an algebraic surface in $\Re^{*}$ having dimension over $F$ equal to $2 m+2 n_{0}\left(n-n_{0}\right)$. (b) The $S B D$ orbits are orbits of maximal dimension in $\mathfrak{C}^{*}$.

Proof. (a) Let $O$ be an SBD orbit represented by $(\lambda, 0,0,0)$ with $\lambda \in \Lambda$. Then $O$ consists of all $\left(t_{1}, y_{1}, x_{1}, w_{1}\right) \in \mathfrak{X}^{*}$ such that $t_{1}=w^{t} \lambda w^{s t}$ for some $w \in W$. By the uniqueness part of Lemma 2.1, $t_{1}$ can be parameterized by $w \in W$. Thus, $\operatorname{dim} O=m+n_{0}\left(n-n_{0}\right)+n_{0}\left(n-n_{0}\right)$ $+m$. (b) The complement of the union of the SBD orbits consists of all elements $\left(t_{1}, y_{1}, x_{1}, w_{1}\right) \in \mathfrak{T}^{*}$ with det $t_{1}^{[k]}=0$ for some $k=1, \ldots, r$. Thus, the union of the SBD orbits is a Zariski open set. It is known that the union of the orbits of maximal dimension is also a Zariski open set. If 
the SBD orbits were not of maximal dimension we would have two disjoint Zariski open sets, which is impossible.

REMARK. We have not proved that there could not be an orbit of maximal dimension which is not SBD.

Now that we have isolated the SBD orbits, we will present the corresponding irreducible unitary representations of $N$. Recall that a subalgebra $\mathcal{Q}$ of the Lie algebra $\Re$ is said to be subordinate to $X^{\prime} \in \Re^{*}$ provided $\left\langle[X, Y] \mid X^{\prime}\right\rangle=0$ for every $X, Y \in \mathcal{Q}$. For fixed $X^{\prime} \in \mathcal{T}^{*}$ let $\mathcal{Q}$ be a subordinate subalgebra of maximal dimension and let $U=\exp$ थ. Define a unitary character on $U$ by setting $\chi(u)=\exp 2 \pi i\left\langle\log u \mid X^{\prime}\right\rangle$, for $u \in U$. The desired irreducible unitary representation of $N$ is the one induced from $\chi$.

It happens that there is a maximal subalgebra subordinate to every $X^{\prime} \in \mathcal{T}^{*}$. In fact, define $\mathscr{U}=\left\{\left(t_{1}, y_{1}, 0,0\right) \in \mathcal{N}\right\}$, then $\mathcal{U}$ is an abelian subalgebra of $\mathcal{K}$ and it can be verified that

$$
\operatorname{dim}_{\mathbf{R}} \mathcal{U}=\left(\operatorname{dim}_{\mathbf{R}} \Re\right)-\left(\operatorname{dim}_{\mathbf{R}} \theta\right) / 2,
$$

for an SBD orbit $\vartheta$, to show that $\mathcal{Q}$ has maximal dimension.

For $X \in \mathcal{Q}$ we have $X^{2}=0$, which implies that $\exp X=I+X$. Let $U=\exp थ$, then $U=\{(t, y, 0, I) \in N\} . U$ is an abelian subgroup of $N$ with group operation

$$
(t, y, 0, I)\left(t^{\prime}, y^{\prime}, 0, I\right)=\left(t+t^{\prime}, y+y^{\prime}, 0, I\right) .
$$

Also, define $V=\{(0,0, x, w) \in N\}$, then $V$ is a subgroup of $N$ with group operation given by

$$
(0,0, x, w)\left(0,0, x^{\prime}, w^{\prime}\right)=\left(0,0, x+w x^{\prime}, w w^{\prime}\right) .
$$

Elements of $N$ can be uniquely expressed in the form $z=u v$, with $u \in U$ and $v \in V$. In fact,

$$
(t, y, x, w)=\left(t w^{s}+y x^{s}, y, 0, I\right)(0,0, x, w) .
$$

Also, $V$ normalizes $U$ since

$$
\begin{aligned}
(0,0, x, w) & (t, y, 0, I)(0,0, x, w)^{-1} \\
= & \left(x y^{s} w^{s}+w t w^{s}+w y x^{s}, w y, 0, I\right)
\end{aligned}
$$

It follows that $N=U V$ is a semidirect product and representations of $N$ induced from characters of $U$ can be realized to act in $L^{2}(V)$.

Fix $\lambda \in \Lambda$, let $X^{\prime}=(\lambda, 0,0,0) \in \Re^{*}$, and consider the unitary character of $U$ defined for $u=(t, y, 0, I) \in U$ by

$$
\chi_{\lambda}(u)=\exp 2 \pi i\left\langle\log u \mid X^{\prime}\right\rangle=\exp 2 \pi i\langle t \mid \lambda\rangle,
$$


where $\langle t \mid \lambda\rangle=\operatorname{Re} \operatorname{tr} t \lambda^{t}$. The irreducible unitary representation of $N$, denoted $\lambda$, induced by $\chi_{\lambda}$ and acting in $L^{2}(V)$ is then given by $\tilde{\lambda}\left(u_{0} v_{0}\right) \phi(v)=\chi_{\lambda}\left(v u_{0} v^{-1}\right) \phi\left(v v_{0}\right)$, for $u_{0} \in U, v_{0} \in V$, and $\phi \in L^{2}(V)$.

Let $X$ be the additive group $F^{\left(n-n_{0}\right) \times n_{0}}$. Choose as independent parameters for the elements $(0,0, x, w) \in V$ the matrices $\xi=w^{-1} x \in X$ and $\mu=w \in W$. The Haar measure of $V$ is $d \xi d \mu$, where $d \xi$ and $d \mu$ denote the Haar measures of $X$ and $W$, so we have an identification of $L^{2}(V)$ with $L^{2}(X \times W)$. In terms of $\xi$ and $\mu$, the formula for $\tilde{\lambda}$ is

$$
\begin{aligned}
& \tilde{\lambda}(t, y, x, w) \phi(\xi, \mu) \\
& =\exp 2 \pi i\left\langle\lambda \mid \mu\left(\xi y^{s}+t w^{s}+y x^{s}+y \xi^{s}\right) \mu^{s}\right\rangle \phi\left(w^{-1}(\xi+x), \mu w\right)
\end{aligned}
$$

for $(t, y, x, w) \in N$ and $\phi \in L^{2}(X \times W)$.

To summarize this application of the theory of Kirillov we state

THEOREM 2.4 (i) For $\lambda \in \Lambda$, (2.23) defines an irreducible unitary representation $\tilde{\lambda}$ of $N$. (ii) The mapping from $\Lambda$ to the dual object of $N$ which sends $\lambda$ to the unitary equivalence class of $\tilde{\lambda}$ is injective, preserves Borel structure, and the complement of the image is a null set with respect to Plancherel measure.

3. The action of $M$ on the dual of $\mathbf{N}$. Since $M$ normalizes $N$, the map $z \rightarrow m^{-1} z m$ is an automorphism of $N$ for each $m \in M$. It follows that $z \rightarrow \tilde{\lambda}\left(m^{-1} z m\right)$, for $\lambda \in \Lambda$, is an irreducible unitary representation of $N$ unitarily equivalent to $(m \cdot \lambda)^{\tilde{n}}$ for some $m \cdot \lambda \in \Lambda$. We now investigate this action $(m, \lambda) \rightarrow m \cdot \lambda$ of $M$ on $\Lambda$.

Denote the elements of $M$, defined in (1.6), by $m=(s, a)$, with $s \in \operatorname{Sp}\left(n_{0}, F\right)$ and $a \in \operatorname{Gl}\left(n_{r}, F\right) \times \cdots \times \operatorname{Gl}\left(n_{1}, F\right)$. Express $s$ in terms of $n_{0} \times n_{0}$ submatrices by writing

$$
s=\left|\begin{array}{ll}
s_{11} & s_{12} \\
s_{21} & s_{22}
\end{array}\right|
$$

and recall that $a$ is regarded as an $\left(n-n_{0}\right) \times\left(n-n_{0}\right)$ block-diagonal matrix. Let $z=(t, y, x, w) \in N$. Since $y, x \in X=F^{\left(n-n_{0}\right) \times n_{0}}$, we can form the $\left(n-n_{0}\right) \times 2 n_{0}$ matrix [yx]. It will be convenient to define

$$
g(y, x)=g([y x])=y x^{s} .
$$

Matrix multiplication now gives the following expression for $m^{-1} \mathrm{zm}$ :

$$
m^{-1} z m=\left(a^{-1} t a^{s-1}, a^{-1}\left(y s_{11}+x s_{21}\right), a^{-1}\left(y s_{12}+x s_{22}\right), a^{-1} w a\right) \text {. }
$$


Let $\lambda \in \Lambda$ and $\phi \in L^{2}(X \times W)$, then (3.2) together with (2.23) yields

$$
\begin{aligned}
& \tilde{\lambda}\left(m^{-1} z m\right) \phi(\xi, \mu) \\
& =\exp 2 \pi i\langle\lambda| \mu\left(\xi\left(y s_{11}+x s_{21}\right)^{s} a^{s-1}\right. \\
& \left.\left.\quad+a^{-1}\left(t w^{s}+g([y x] s)\right) a^{s-1}+a^{-1}\left(y s_{11}+x s_{21}\right) \xi^{s}\right) \mu^{s}\right\rangle \\
& \quad \cdot \phi\left(a^{-1} w^{-1} a\left(\xi+a^{-1}\left(y s_{12}+x s_{22}\right)\right), \mu a^{-1} w a\right) .
\end{aligned}
$$

Since the representation $z \rightarrow \tilde{\lambda}\left(m^{-1} z m\right)$ is unitarily equivalent to $(m \cdot \lambda)^{\sim}$, there exists a unitary operator $D_{\lambda}(m)$ on $L^{2}(X \times W)$, for each $m \in M$ and $\lambda \in \Lambda$, such that

$$
\tilde{\lambda}\left(m^{-1} z m\right)=D_{\lambda}(m)^{-1}(m \cdot \lambda)^{\tilde{}}(z) D_{\lambda}(m),
$$

for all $z \in N$. The operator $D_{\lambda}(m)$ is unique up to a scalar factor in $\mathcal{J}=\{\alpha \in \mathbf{C}:|\alpha|=1\}$, because $(m \cdot \lambda)^{\sim}$ is irreducible. Also, for $m_{1}$, $m_{2} \in M$,

$$
\tilde{\lambda}\left(m_{2}^{-1}\left(m_{1}^{-1} z m_{1}\right) m_{2}\right)=\tilde{\lambda}\left(\left(m_{1} m_{2}\right)^{-1} z\left(m_{1} m_{2}\right)\right)
$$

implies that $m_{1} \cdot\left(m_{2} \cdot \lambda\right)=\left(m_{1} m_{2}\right) \cdot \lambda$, from which it follows

$$
D_{\lambda}\left(m_{1} m_{2}\right)=\alpha\left(\lambda, m_{1}, m_{2}\right) D_{m_{2} . \lambda}\left(m_{1}\right) D_{\lambda}\left(m_{2}\right),
$$

for some $\alpha\left(\lambda, m_{1}, m_{2}\right) \in \mathcal{T}$.

The way to describe the operators $D_{\lambda}(m)$ and the action $(m, \lambda) \rightarrow m \cdot \lambda$ is to work separately with the following obvious subgroups of $M$ :

$$
S=\left\{(s, I): s \in \operatorname{Sp}\left(n_{0}, F\right)\right\}
$$

and

$$
A=\left\{(I, a): a \in \mathrm{Gl}\left(n_{r}, F\right) \times \cdots \times \mathrm{Gl}\left(n_{1}, F\right)\right\} .
$$

Fix $\lambda \in \Lambda$. It is easy to see from (3.2) that $A$ normalizes the subgroup $V=\{(0,0, x, w) \in N\}$ of $N$. Therefore, the formula

$$
D_{\lambda}(m) \phi(v)=\gamma(m)^{1 / 2} \phi\left(m^{-1} v m\right)
$$

$\left(m \in A, \phi \in L^{2}(V)\right)$, where $\gamma(m)=d\left(m^{-1} v m\right) / d v$, defines a unitary operator on $L^{2}(V)$. If $m=(I, a) \in A$ then, in terms of the parameters $(\xi, \mu)$, the formula becomes

$$
D_{\lambda}(I, a) \phi(\xi, \mu)=\gamma(I, a)^{1 / 2} \phi\left(a^{-} \xi, a^{-1} \mu a\right),
$$


for $\phi \in L^{2}(X \times W)$. A simple computation shows

Lemma 3.1. For $m=(I, a) \in A$ and $\lambda \in \Lambda$,

$$
\tilde{\lambda}\left(m^{-1} z m\right)=D_{\lambda}(m)^{-1}\left(a^{t-1} \lambda a^{s t-1}\right)^{\tilde{}}(z) D_{\lambda}(m),
$$

for all $z \in N, D_{\lambda}(m)$ defined by (3.6). Thus, $m \cdot \lambda=a^{t-1} \lambda a^{s t-1}$.

If $n_{0}=0$ then $M=A$ and we are done, so assume in the discussion of $S$ that follows that $n_{0} \neq 0$. Now let $m=(s, I) \in S$. In order to describe $D_{\lambda}(s, I)$, we need to parallel Weil's construction of the oscillator representation (cf. [4] 2.3). Fix $\lambda \in \mathrm{Gl}\left(n-n_{0}, F\right)$ and let $X$ be the vector space $F^{\left(n-n_{0}\right)} \times n_{0}$ over $F$. The dual of the additive group of $X$ is identified with $X$ using the bicharacter

$$
\langle x, y\rangle_{\lambda}=\exp 2 \pi i\left\langle\lambda \mid x y^{s}+y x^{s}\right\rangle=\chi_{\lambda}\left(x y^{s}+y x^{s}\right) .
$$

For each $(y, x) \in X \times X$, let $U_{\lambda}^{\prime}(y, x)$ denote the unitary operator on $L^{2}(X)$ defined by

$$
U_{\lambda}^{\prime}(y, x) \psi(\xi)=\chi_{\lambda}\left(\xi y^{s}+y \xi^{s}\right) \psi(\xi+x) .
$$

The family of operators

$$
U_{\lambda}(y, x, \alpha)=\alpha U_{\lambda}^{\prime}(y, x) \quad(y, x \in X, \alpha \in \mathcal{T})
$$

forms a group, denoted $\overline{A_{\lambda}(X)}$, whose composition law is

$$
\begin{aligned}
& U_{\lambda}\left(y_{1}, x_{1}, \alpha_{1}\right) U_{\lambda}\left(y_{2}, x_{2}, \alpha_{2}\right) \\
& =U_{\lambda}\left(y_{1}+y_{2}, x_{1}+x_{2}, \chi_{\lambda}\left(y_{2} x_{1}^{s}+x_{1} y_{2}^{s}\right) \alpha_{1} \alpha_{2}\right) .
\end{aligned}
$$

There is an isomorphic group $A_{\lambda}(X)=X \times X \times \mathcal{T}$ with composition law

$$
\left(y_{1}, x_{1}, \alpha_{1}\right)\left(y_{2}, x_{2}, \alpha_{2}\right)=\left(y_{1}+y_{2}, x_{1}+x_{2}, x_{\lambda}\left(y_{2} x_{1}^{s}+x_{1} y_{2}^{s}\right) \alpha_{1} \alpha_{2}\right) \text {. }
$$

$U_{\lambda}: A_{\lambda}(X) \rightarrow \overline{A_{\lambda}(X)}$ is the unique irreducible unitary representation of $A_{\lambda}(X)$ which leaves the center, $\{0\} \times\{0\} \times \mathcal{T}$, pointwise fixed, in the sense that $U_{\lambda}(0,0, \alpha)=\alpha I$.

Let $B_{\lambda}(X)$ denote the group of automorphisms of $A_{\lambda}(X)$ which leave the center pointwise fixed and let $\sigma \in B_{\lambda}(X)$. Then there exists a unitary operator $r_{\lambda}(\sigma)$ of $L^{2}(X)$, unique up to a scalar factor in $\mathcal{T}$, such that

$$
U_{\lambda}((y, x, \alpha) \sigma)=r_{\lambda}(\sigma)^{-1} U_{\lambda}(y, x, \alpha) r_{\lambda}(\sigma) .
$$

The mapping $\sigma \rightarrow r_{\lambda}(\sigma)$ determines a projective representation of $B_{\lambda}(X)$ called the Weil representation.

Let $s \in \operatorname{Sp}\left(n_{0}, F\right)$ and define $f_{s, \lambda}: X \times X \rightarrow \mathcal{T}$ by

$$
f_{s, \lambda}(y, x)=\chi_{\lambda}(g([y x] s)-g([y x]),
$$


where $g([y x])=y x^{s}$. Then the pair $\left(s, f_{s, \lambda}\right)$ determines an element of $B_{\lambda}(X)$ defined by

$$
(y, x, \alpha)\left(s, f_{s, \lambda}\right)=\left([y x] s, f_{s, \lambda}(y, x) \alpha\right),
$$

for $(y, x, \alpha) \in A_{\lambda}(X)$, as a computation shows. Morever, the set $\left\{\left(s, f_{s, \lambda}\right)\right.$ : $\left.s \in \operatorname{Sp}\left(n_{0}, F\right)\right\}$ together with the operation

$$
\left(s_{1}, f_{s_{1}, \lambda}\right)\left(s_{2}, f_{s_{2}, \lambda}\right)=\left(s_{1} s_{2}, f_{s_{1} s_{2}, \lambda}\right)
$$

forms a subgroup of $B_{\lambda}(X)$ isomorphic to $\operatorname{Sp}\left(n_{0}, F\right)$. For each $s \in$ $\operatorname{Sp}\left(n_{0}, F\right)$, use $\sigma=\left(s, f_{s, \lambda}\right)$ in (3.9) to define

$$
r_{\lambda}(s)=r_{\lambda}\left(s, f_{s, \lambda}\right) \text {. }
$$

The mapping $s \rightarrow r_{\lambda}(s)$ determines a projective representation of $\operatorname{Sp}\left(n_{0}, F\right)$; this is the oscillator representation.

In order to make use of $r_{\lambda}$ in the context of the representation $\tilde{\lambda}$ of $N$ acting in $L^{2}(X \times W)$, we identify $L^{2}(X \times W)$ with $L^{2}\left(W, L^{2}(X)\right)$. That is, the function $\phi \in L^{2}(X \times W)$ is identified with the $L^{2}(X)$-valued function

$$
\mu \rightarrow \phi_{\mu}=\phi(\cdot, \mu),
$$

defined for $\mu \in W$. Recall that an operator $Q$ on $L^{2}\left(W, L^{2}(X)\right)$ is called decomposable if $(Q \phi)_{\mu}=Q(\mu) \phi_{\mu}$ for $\phi \in L^{2}\left(W, L^{2}(X)\right)$, where $Q(\mu)$ is an operator on $L^{2}(X)$ for each $\mu \in W$. In this case we say $Q=\int_{W} Q(\mu) d \mu$. If we define $N_{1}=\{(t, y, x, I) \in N\}$, then $N_{1}$ is a subgroup of $N$ and the operators $\tilde{\lambda}(t, y, x, I)$ are decomposable. For $\lambda \in \Lambda$ and $\mu \in W$, we will use the notation

$$
\left\{\begin{array}{l}
\lambda \cdot \mu=\mu^{t} \lambda \mu^{s t} \\
(\tilde{\lambda} \cdot \mu)(t, y, x, I)=\chi_{\lambda \cdot \mu}\left(t+y x^{s}\right) U_{\lambda \cdot \mu}^{\prime}(y, x) .
\end{array}\right.
$$

Then (2.23) and (3.8) imply

$$
\tilde{\lambda}(t, y, x, I)=\int_{W}(\tilde{\lambda} \cdot \mu)(t, y, x, I) d \mu
$$

The relationship between $\tilde{\lambda}$ and the oscillator representation is given by the following sequence of results:

Lemma 3.2. Let $\lambda \in \Lambda, \mu \in W,(t, y, x, I) \in N_{1}$, and $s \in \operatorname{Sp}\left(n_{0}, F\right)$, then

$$
(\tilde{\lambda} \cdot \mu)(t,[y x] s, I)=r_{\lambda \cdot \mu}(s)^{-1}(\tilde{\lambda} \cdot \mu)(t, y, x, I) r_{\lambda \cdot \mu}(s)
$$


Proof. Here is an outline of the proof. (1) Define $\tau_{\lambda \cdot \mu}: N_{1} \rightarrow A_{\lambda \cdot \mu}(X)$ by the formula

$$
\tau_{\lambda \cdot \mu}(t, y, x, I)=\left(y, x, \chi_{\lambda \cdot \mu}\left(t+y x^{s}\right)\right),
$$

then $\tau_{\lambda \cdot \mu}$ is a homomorphism and $\tilde{\lambda} \cdot \mu=U_{\lambda \cdot \mu} \tau_{\lambda \cdot \mu}$. (2) Denote by $\overline{(s, I)}$ the automorphism of $N_{1}$ given by

$$
(t, y, x, I) \rightarrow(t,[y x] s, I) .
$$

Then the following is a commutative diagram:

$$
\begin{array}{ccc}
N_{1} & \overline{(s, I)} & N_{1} \\
\tau_{\lambda \mu} \downarrow & & \downarrow \tau_{\lambda \cdot \mu} \\
A_{\lambda \cdot \mu}(X) & \underset{\left(s, \overrightarrow{f_{s, \lambda}, \mu}\right)}{\rightarrow} & A_{\lambda \cdot \mu}(X)
\end{array}
$$

(3) Denote by $\overline{r_{\lambda \cdot \mu}(s)}$ the automorphism of $\overline{A_{\lambda \cdot \mu}(X)}$ given by

$$
U_{\lambda \cdot \mu}(y, x, \alpha) \rightarrow r_{\lambda \cdot \mu}(s)^{-1} U_{\lambda \cdot \mu}(y, x, \alpha) r_{\lambda \cdot \mu}(s),
$$

then the following is a commutative diagram:

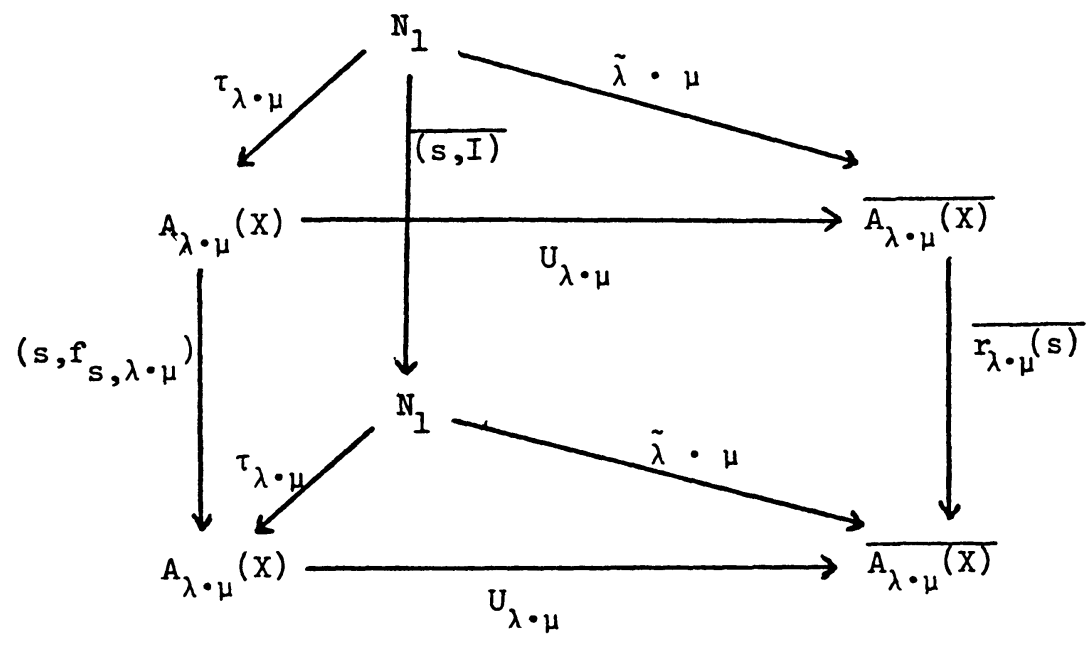

which completes the proof.

LEMMA 3.3. For $\mu \in W$, define a unitary operator $l(\mu)$ on $L^{2}(X)$ by $l(\mu) \psi(\xi)=\psi\left(\mu^{-1} \xi\right)$. Then, for $s \in \operatorname{Sp}\left(n_{0}, F\right)$,

$$
r_{\lambda \cdot \mu}(s)=l(\mu)^{-1} r_{\lambda}(s) l(\mu),
$$

up to a scalar factor in $\mathcal{T}$. 
Proof. First, note that for $\psi \in L^{2}(X)$ and $(t, y, x, I) \in N_{1}$,

$$
\begin{aligned}
& l(\mu)^{-1} \chi_{\lambda}\left(\mu\left(t+y x^{s}\right) \mu^{s}\right) U_{\lambda}^{\prime}(\mu[y x]) l(\mu) \psi(\xi) \\
& \quad=\chi_{\lambda}\left(\mu\left(t+y x^{s}\right) \mu^{s}\right) \chi_{\lambda}\left(\mu\left(\xi y^{s}+y \xi^{s}\right) l(\mu) \psi(\mu \xi+\mu x)\right. \\
& \quad=\chi_{\lambda \cdot \mu}\left(t+y x^{s}\right) \chi_{\lambda \cdot \mu}\left(\xi y^{s}+y \xi^{s}\right) \chi(\xi+x) \\
& \quad=\chi_{\lambda \cdot \mu}\left(t+y x^{s}\right) U_{\lambda \cdot \mu}^{\prime}(y, x) \psi(\xi)
\end{aligned}
$$

That is,

$$
l(\mu)^{-1} \tilde{\lambda} \cdot I\left(\mu t \mu^{s}, \mu[y x], I\right) l(\mu)=\tilde{\lambda} \cdot \mu(t, y, x, I) .
$$

It follows that

$$
\begin{aligned}
l(\mu)^{-1} & r_{\lambda}(s)^{-1} l(\mu) \tilde{\lambda} \cdot \mu(t, y, x, I) l(\mu)^{-1} r_{\lambda}(s) l(\mu) \\
& =l(\mu)^{-1} r_{\lambda}(s)^{-1} \tilde{\lambda} \cdot I\left(\mu t \mu^{s}, \mu[y x], I\right) r_{\lambda}(s) l(\mu) \\
& =l(\mu)^{-1} \tilde{\lambda} \cdot I\left(\mu t \mu^{s}, \mu[y x] s, I\right) l(\mu) \\
& =\tilde{\lambda} \cdot \mu(t,[y x] s, I),
\end{aligned}
$$

using (3.16) and (3.15). Since $r_{\lambda \cdot \mu}(s)$ is the unique unitary operator (up to scalar factor in $\mathscr{T}$ ) with this property, we have the desired result.

TheOREM 3.3. For $(s, I) \in S$, define the unitary operator $D_{\lambda}(s)$ on $L^{2}\left(W, L^{2}(X)\right)$ by setting

$$
D_{\lambda}(s)=\int_{W} l(\mu)^{-1} r_{\lambda}(s) l(\mu) d \mu
$$

Then,

$$
\tilde{\lambda}\left((s, I)^{-1}(t, y, x, w)(s, I)\right)=D_{\lambda}(s)^{-1} \tilde{\lambda}(t, y, x, w) D_{\lambda}(s),
$$

for every $(t, y, x, w) \in N$.

Proof. For $(t, y, x, w) \in N$, we have

$$
\tilde{\lambda}(t, y, x, w)=\tilde{\lambda}\left(t w^{s}, y, x, I\right) \tilde{\lambda}(0,0,0, w)
$$

and, hence,

$$
\begin{aligned}
& D_{\lambda}(s)^{-1} \tilde{\lambda}(t, y, x, w) D_{\lambda}(s) \\
& \quad=D_{\lambda}(s)^{-1} \tilde{\lambda}\left(t w^{s}, y, x, I\right) D_{\lambda}(s) D_{\lambda}(s)^{-1} \tilde{\lambda}(0,0,0, w) D_{\lambda}(s) .
\end{aligned}
$$


But, for $\phi \in L^{2}\left(W, L^{2}(X)\right)$,

$$
\begin{aligned}
& \left(D_{\lambda}(s)^{-1} \tilde{\lambda}\left(t w^{s}, y, x, I\right) D_{\lambda}(s) \phi\right)_{\mu}(\xi) \\
& \quad=l(\mu)^{-1} r_{\lambda}(s)^{-1} l(\mu) \tilde{\lambda} \cdot \mu\left(t w^{s}, y, x, I\right) l(\mu)^{-1} r_{\lambda}(s) l(\mu) \phi_{\mu}(\xi) \\
& \quad=r_{\lambda \cdot \mu}(s)^{-1} \tilde{\lambda} \cdot \mu\left(t w^{s}, y, x, I\right) r_{\lambda \cdot \mu}(s) \phi_{\mu}(\xi) \\
& \quad=\tilde{\lambda} \cdot \mu\left((s, I)^{-1}\left(t w^{s}, y, x, I\right)(s, I)\right) \phi_{\mu}(\xi) \\
& \quad=\left(\tilde{\lambda}\left((s, I)^{-1}\left(t w^{s}, y, x, I\right)(x, I)\right) \phi\right)_{\mu}(\xi),
\end{aligned}
$$

by Lemma 3.3 and (3.15). Also, from (2.23), we have

$$
(\tilde{\lambda}(0,0,0, w) \phi)_{\mu}(\xi)=\phi_{\mu w}\left(w^{-1} \xi\right)=l(w) \phi_{\mu w}(\xi) .
$$

Thus,

$$
\begin{aligned}
& \left(D_{\lambda}(s)^{-1} \tilde{\lambda}(0,0,0, w) D_{\lambda}(s) \phi\right)_{\mu}(\xi) \\
& \quad=l(\mu)^{-1} r_{\lambda}(s)^{-1} l(\mu)\left(\tilde{\lambda}(0,0,0, w) D_{\lambda}(s) \phi\right)_{\mu}(\xi) \\
& \quad=l(\mu)^{-1} r_{\lambda}(s)^{-1} l(\mu) l(w)\left(D_{\lambda}(s) \phi\right)_{\mu w}(\xi) \\
& \quad=l(\mu)^{-1} r_{\lambda}(s)^{-1} l(\mu) l(w) l(\mu w)^{-1} r_{\lambda}(s) l(\mu w) \phi_{\mu w}(\xi) \\
& \quad=l(w) \phi_{\mu w}(\xi)=(\tilde{\lambda}(0,0,0, w) \phi)_{\mu}(\xi)
\end{aligned}
$$

that is, $\tilde{\lambda}(0,0,0, w)$ commutes with $D_{\lambda}(s)$. Combining these facts yields

$$
\begin{aligned}
& D_{\lambda}(s)^{-1} \tilde{\lambda}(t, y, x, w) D_{\lambda}(s) \\
&= D_{\lambda}(s)^{-1} \tilde{\lambda}\left(t w^{s}, y, x, I\right) \tilde{\lambda}(0,0,0, w) D_{\lambda}(s) \\
&=D_{\lambda}(s)^{-1} \tilde{\lambda}\left(t w^{s}, y, x, I\right) D_{\lambda}(s) \tilde{\lambda}(0,0,0, w) \\
&=\tilde{\lambda}\left((s, I)^{-1}\left(t w^{s}, y, x, I\right)(s, I)\right) \tilde{\lambda}(0,0,0, w) \\
&=\tilde{\lambda}\left(t w^{s},[y x] s, I\right) \tilde{\lambda}(0,0,0, w) \\
&=\tilde{\lambda}(t,[y x] s, w)=\tilde{\lambda}\left((s, I)^{-1}(t, y, x, w)(s, I)\right) .
\end{aligned}
$$

In summary, we have the result:

THEOREM 3.4 (a) The action of $M$ on $\Lambda$ is given by

$$
(s, a) \cdot \lambda=a^{t-1} \lambda a^{s t-1} \quad \text { for }(s, a) \in M, \lambda \in \Lambda .
$$
have

(b) With $D_{\lambda}(s, a)=D_{\lambda}(s, I) D_{\lambda}(I, a)$ for $\lambda \in \Lambda$ and $(s, a) \in M$, we

$$
\tilde{\lambda}\left((s, a)^{-1} z(s, a)\right)=D_{\lambda}(s, a)^{-1}\left(a^{t-1} \lambda a^{s t-1}\right)^{\tilde{}}(z) D_{\lambda}(s, a),
$$

for all $z \in N$. 
Next, we investigate the orbits in $\Lambda$ under the action of $M$. Since (3.19) is correct for $n_{0}=0$ as well as for $n_{0} \neq 0$, the results here hold in both cases. For any $\lambda \in \Lambda$, let $\lambda=\left(\lambda^{(1)}, \ldots, \lambda^{(r)}\right)$, where $\lambda^{(k)}=\left(\lambda^{(k)}\right)^{s}$ is the non-singular, $n_{k} \times n_{k}$ submatrix of $\lambda$ in the $k$ th position $(k=1, \ldots, r)$ along the secondary block-diagonal of $\lambda$ (counting from lower left to upper right). For $(s, a) \in M$, let $a=\left(a^{(1)}, \ldots, a^{(r)}\right)$, where $a^{(k)} \in$ $\operatorname{Gl}\left(n_{k}, F\right)$ is the submatrix of $a$ in the $k$ th position along the block-diagonal (counting from lower right to upper left). In terms of this notation, the action of $M$ on $\Lambda$ is given by

$$
m \cdot \lambda=\left(a^{(1) t-1} \lambda^{(1)} a^{(1) s t-1}, \ldots, a^{(r) t-1} \lambda^{(r)} a^{(r) s t-1}\right) .
$$

A variant of Sylvestor's Law can be applied to each component to prove the following result:

THEOREM 3.5. For $F=\mathbf{C}$, the action of $M$ on $\Lambda$ is transitive. For $F=\mathbf{R}$, there are $\left(n_{1}+1\right)\left(n_{2}+1\right) \cdots\left(n_{r}+1\right)$ orbits in $\Lambda$ under the action of $M$. Each orbit is represented by an element $\left(\lambda^{(1)}, \ldots, \lambda^{(r)}\right)$ such that, for $k=1, \ldots, r, \lambda^{(k)}$ has the secondary diagonal form

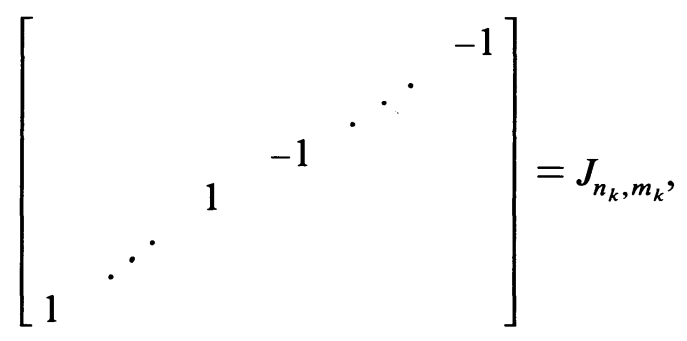

with $m_{k}$ entries of 1 in the lower left positions and $n_{k}-m_{k}$ entries of -1 in the remaining positions $\left(m_{k}=0,1, \ldots, n_{k}\right)$.

4. The commuting algebra of $T$ restricted to $N M$. Corresponding to a certain blocking scheme for $2 n \times 2 n$ matrices, we have defined a parabolic subgroup $P$ of $\operatorname{Sp}(n, F)$, the opposite parabolic $N M$, and the principal series representation $T$ induced from a unitary character on $P$ and acting in $L^{2}(N)$. Next, we obtain theorems concerning the commuting algebra of $\left.T\right|_{N M}$ generalizing results in [6], [2], and [1]. Proofs will be omitted here; they are similar to what can be found in the papers just mentioned.

Let $\Lambda_{1}, \ldots, \Lambda_{l}$ denote the orbits in $\Lambda$ under the action of $A$ and let $\lambda_{1}, \ldots, \lambda_{l}$ be orbit representatives (see Theorem 3.5 ). Define the stability subgroup of $\lambda_{i}$ in $A$ :

$$
A_{i}=\left\{(I, a) \in A:(I, a) \cdot \lambda_{i}=\lambda_{i}\right\},
$$

for $i=1, \ldots, l$. The subgroup $A_{i}$ is uniquely determined by the orbit, up to conjugate subgroups in $A$. We are also interested in the stability subgroup 
of $\lambda_{i}$ in $M$. This turns out to be $S A_{i}$, as is easily seen. Consider, now, the restriction of $D_{\lambda_{i}}$ to $S A_{i}$. By (3.5), this is a multiplier representation of $S A_{i}$ acting in $L^{2}(X \times W)$. The two theorems below describe the stability subgroups and $D_{\lambda_{i} \mid S A_{i}}$.

Analogous to the notation $J_{n, m}$ of Theorem 3.5, we have

$$
I_{n, m}=\left[\begin{array}{ll}
-I_{n-m} & 0 \\
0 & I_{m}
\end{array}\right] \quad(m=0,1, \ldots, n),
$$

where $I_{m}$ and $I_{n-m}$ denote identity matrices. The (pseudo) orthogonal groups are defined by

$$
O(m, n-m, F)=\left\{a \in \mathrm{Gl}(n, F): a I_{n, m} a^{t}=I_{n, m}\right\} .
$$

If $m=0$ then $O(0, n-0, F)=O(n, F)$ is the usual orthogonal group.

TheOREM 4.1. Let $F=\mathbf{C}$. The single orbit $\Lambda_{1}=\Lambda$ is represented by $\lambda_{1}=\left(J_{n_{1}}, \ldots, J_{n_{r}}\right)=J_{n-n_{0}}$ and $A_{1}$ is isomorphic to

$$
O\left(n_{1}, \mathbf{C}\right) \times \cdots \times O\left(n_{r}, \mathbf{C}\right) .
$$

If $n_{0}=0$, then $S A_{1}=A_{1}$ and $D_{\lambda_{1} \mid S A_{1}}$ is given by

$$
D_{\lambda_{1}}(a) \phi(\mu)=\phi\left(a^{-1} \mu a\right),
$$

for $\phi \in L^{2}(W)$ and $a \in O\left(n_{1}, \mathbf{C}\right) \times \cdots \times O\left(n_{r}, \mathbf{C}\right)$. If $n_{0} \neq 0$, then $S A_{1}$ is isomorphic to

$$
\operatorname{Sp}\left(n_{0}, \mathbf{C}\right) \times O\left(n_{1}, \mathbf{C}\right) \times \cdots \times O\left(n_{r}, \mathbf{C}\right)
$$

and $D_{\lambda_{1} \mid S A_{1}}$ is given by

$$
\left(D_{\lambda_{1}}(s, a) \phi\right)_{\mu}(\xi)=l(\mu)^{-1} r_{\lambda_{1}}(s) l(\mu) l(a) \phi_{a^{-1} \mu a}(\xi),
$$

for $\phi \in L^{2}\left(W, L^{2}(X)\right), s \in \operatorname{Sp}\left(n_{0}, \mathbf{C}\right)$, and $a \in O\left(n_{1}, \mathbf{C}\right) \times \cdots \times O\left(n_{r}, \mathbf{C}\right)$.

REMARK. The description of $A_{1}$ is due to the following: for $a=$ $\left(a^{(1)}, \ldots, a^{(r)}\right) \in \mathrm{Gl}\left(n_{1}, \mathrm{C}\right) \times \cdots \times \mathrm{Gl}\left(n_{r}, \mathbf{C}\right)$,

$$
a^{t-1} J a^{s t-1}=J \Leftrightarrow a^{(k) t-1} J_{n_{k}} a^{(k) s t-1}=J_{n_{k}} \text { for all } k=1, \ldots, r .
$$

But multiplying both sides of this equation by $J_{n_{k}}$ on the right results in $a^{(k) t-1} a^{(k)-1}=I_{n_{k}}$. Thus, $(I, a) \in A_{1}$ if and only if $a^{(k) t-1} \in O\left(n_{k}, \mathrm{C}\right)$ for all $k=1, \ldots, r$. This means $a^{(k)} \in O\left(n_{k}, \mathbf{C}\right)$ for all $k$.

THeOREM 4.2. Let $F=\mathbf{R}$. Let $\Lambda_{1}$ be the orbit represented by $\lambda_{1}=$ $\left(J_{n_{1}, m_{1}}, \ldots, J_{n_{r}, m_{r}}\right)$. Then $A_{1}$ is isomorphic to

$$
O\left(m_{1}, n_{1}-m_{1}, \mathbf{R}\right) \times \cdots \times O\left(m_{r}, n_{r}-m_{r}, \mathbf{R}\right) .
$$


If $n_{0}=0$, then $S A_{1}=A_{1}$ and $D_{\lambda_{1} \mid S A_{1}}$ is given by

$$
D_{\lambda_{1}}(a) \phi(\mu)=\phi\left(a^{-1} \mu a\right),
$$

for $\phi \in L^{2}(W)$ and $a \in O\left(m_{1}, n_{1}-m_{1}, \mathbf{R}\right) \times \cdots \times O\left(m_{r}, n_{r}-m_{r}, \mathbf{R}\right)$. If $n_{0} \neq 0$, then $S A_{1}$ is isomorphic to

$$
\operatorname{Sp}\left(n_{0}, \mathbf{R}\right) \times O\left(m_{1}, n_{1}-m_{1}, \mathbf{R}\right) \times \cdots \times O\left(m_{r}, n_{r}-m_{r}, \mathbf{R}\right)
$$

and $D_{\lambda_{1} \mid S A_{1}}$ is given by

$$
\left(D_{\lambda_{1}}(s, a) \phi\right)_{\mu}(\xi)=l(\mu)^{-1} r_{\lambda_{l}}(s) l(\mu) l(a) \phi_{a^{-1} \mu a}(\xi),
$$

for $\phi \in L^{2}\left(W, L^{2}(X)\right)$.

Corresponding to the orbit representatives $\lambda_{1}, \ldots, \lambda_{l}$, let $Q_{i}^{\prime}=$ $\mathbb{Q}^{\prime}\left(D_{\lambda_{1} \mid S A_{i}}\right)$ denote the commuting algebra of the multiplier representation of $S A_{i}(i=1, \ldots, l)$. Let $L_{i} \in \mathbb{Q}_{i}^{\prime}$, then

$$
(I, a) \cdot \lambda_{i} \rightarrow D_{\lambda_{i}}(I, a) L_{i} D_{\lambda_{i}}(I, a)^{-1},
$$

for $(I, a) \in A$, is a well-defined map from $\Lambda_{i}$ into $\mathcal{L}\left(L^{2}(V)\right)$. For suppose $(I, a) \cdot \lambda_{i}=\left(I, a^{\prime}\right) \cdot \lambda_{i}$, then $\left(I, a^{\prime}\right)^{-1}(I, a)=\left(I, a^{\prime \prime}\right) \in A_{i}$ and $(I, a)=$ $\left(I, a^{\prime}\right)\left(I, a^{\prime \prime}\right)$. Thus,

$$
\begin{aligned}
D_{\lambda_{i}}(I, a) L_{i} D_{\lambda_{i}}(I, a)^{-1} & =D_{\lambda_{i}}\left(I, a^{\prime}\right) D_{\lambda_{i}}\left(I, a^{\prime \prime}\right) L_{i} D_{\lambda_{i}}\left(I, a^{\prime \prime}\right)^{-1} D_{\lambda_{i}}\left(I, a^{\prime}\right)^{-1} \\
& =D_{\lambda_{l}}\left(I, a^{\prime}\right) L_{i} D_{\lambda_{i}}\left(I, a^{\prime}\right)^{-1}
\end{aligned}
$$

since $L_{i}$ commutes with $D_{\lambda_{i}}\left(I, a^{\prime \prime}\right)$.

We can now state the main results regarding the commuting algebra $\mathcal{Q}^{\prime}\left(\left.T\right|_{N M}\right)$. Let $\mathscr{P}: L^{2}(N) \rightarrow L^{2}\left(\Lambda, H S\left(L^{2}(V)\right), d m(\lambda)\right)$ be the Plancherel transform of $L^{2}(N)$ defined as in [6]. Let $\hat{T}=\mathscr{P} T \mathscr{P}^{-1}$.

THEOREM 4.3. Let $F=$ C. Let $\lambda_{1}, A_{1}$, and $D_{\lambda_{1}}$ be as in Theorem 4.2. The mapping from $\mathbb{Q}^{\prime}\left(D_{\left.\lambda_{1} \mid S A_{1}\right)}\right.$ to $\mathcal{L}\left(L^{2}\left(\Lambda, H S\left(L^{2}(V)\right)\right.\right.$, dm $\left.\left.(\lambda)\right)\right)$ defined by

$$
L_{1} \rightarrow \int_{\Lambda} D_{\lambda_{1}}(I, a) L_{1} D_{\lambda_{1}}(I, a)^{-1} \otimes I d m\left((I, a) \cdot \lambda_{1}\right)
$$

$(a \in A)$ is $a^{*}$-isomorphism between the von Neumann algebras $\mathbb{Q}^{\prime}\left(D_{\lambda_{1} \mid S A_{1}}\right)$ and $\mathbb{Q}^{\prime}\left(\left.\hat{T}\right|_{N M}\right)$. Thus, $\mathbb{Q}^{\prime}\left(\left.T\right|_{N M}\right)$ is ${ }^{*}$-isomorphic with $\mathbb{Q}^{\prime}\left(D_{\lambda_{1} \mid S A_{1}}\right)$.

Proof. This follows from [3] (Theorem 6) along with Theorem 4.1. 
Similarly, we have

TheOREM 4.4. Let $F=\mathbf{R}$. Let $\lambda_{1}, \ldots, \lambda_{l}$ be the orbit representatives given in Theorem 3.5, where $l=\left(n_{1}+1\right) \cdots\left(n_{r}+1\right)$. Let $A_{i}$ and $D_{\lambda_{i}}$ $(i=1, \ldots, l)$ correspond to $\lambda_{i}$ as in Theorem 4.2. The mapping from $\mathbb{Q}_{1}^{\prime} \oplus \cdots \oplus \mathbb{Q}_{l}^{\prime}$ to $\mathcal{L}\left(L^{2}\left(\Lambda, H S\left(L^{2}(V)\right), d m(\lambda)\right)\right)$ defined by

$$
\left(L_{1}, \ldots, L_{l}\right) \rightarrow \bigoplus_{i=1}^{l} \int_{\Lambda_{l}} D_{\lambda_{l}}(I, a) L_{i} D_{\lambda_{l}}(I, a)^{-1} \otimes I d m\left((I, a) \cdot \lambda_{i}\right),
$$

$(a \in A)$ is $a^{*}$-isomorphism between the von Neumann algebras $\mathbb{Q}_{1}^{\prime}$ $\oplus \cdots \oplus \mathbb{Q}^{\prime}$ and $\mathbb{Q}^{\prime}\left(\left.\hat{T}\right|_{N M}\right)$. Thus, $\mathbb{Q}^{\prime}\left(\left.T\right|_{N M}\right)$ is ${ }^{*}$-isomorphic with $\mathbb{Q}^{\prime}\left(D_{\lambda_{1} \mid S A_{1}}\right)$ $\oplus \cdots \oplus \mathbb{Q}^{\prime}\left(D_{\lambda_{l} \mid S A_{l}}\right)$.

\section{REFERENCES}

[1] T. Farmer, On the reduction of certain degenerate principal series representations of $\mathrm{Sp}(n$, C), Pacific. J. Math., 84, No. 2, (1979), 291-303.

[2] - Irreducibility of certain degenerate principal series representations of $\operatorname{Sp}(n, \mathbf{R})$, Proc. Amer. Math. Soc., 83, No. 2, (1981), 411-420.

[3] S. Gaal, Linear Analysis and Representation Theory, New York, Heidelberg, Berlin, Springer, 1973.

[4] S. Gelbart, Weil's Representation and the Spectrum of the Metaplectic Group, Springer Lecture Notes, No. 530, 1976.

[5] I. M. Gelfand and M. A. Naimark, Unitäre Darstellungen der Klassischen Gruppen, Berlin, Akadamie 1957.

[6] K. I. Gross, The dual of a parabolic subgroup and a degenerate principal series of $\operatorname{Sp}(n$, C), Amer. J. Math., 93 (1971), 398-428.

[7] A. A. Kirillov, Unitary representations of nilpotent Lie groups, Russian Math. Surveys, 17 (1962), 53-104.

[8] J. A. Wolf, Unitary representations of maximal parabolic subgroups of the classical groups, Amer. Math. Soc. Memoir, 180 (1976).

Received October 14, 1981.

MiAmi University OXFORD, OH 45056 


\title{
PACIFIC JOURNAL OF MATHEMATICS \\ EDITORS
}

\author{
DONALD BABBITT (Managing Editor) \\ University of California \\ Los Angeles, CA 90024 \\ Hugo Rossi \\ University of Utah \\ Salt Lake City, UT 84112 \\ C. C. Moore and Arthur Ogus \\ University of California \\ Berkeley, CA 94720
}

\author{
J. DugundiI \\ Department of Mathematics \\ University of Southern California \\ Los Angeles, CA 90089-1113
}

R. FINN and H. SAMELSON

Stanford University

Stanford, CA 94305

\begin{tabular}{lllll}
\multicolumn{3}{c}{ ASSOCIATE EDITORS } \\
R. ARENS & $\begin{array}{lll}\text { E. F. BeCKendach } \\
(1906-1982)\end{array}$ & B. H. NeumanN & F. Wolf & K. Yoshida
\end{tabular}

\begin{tabular}{ll}
\multicolumn{2}{c}{ SUPPORTING INSTITUTIONS } \\
UNIVERSITY OF ARIZONA & UNIVERSITY OF OREGON \\
UNIVERSITY OF BRITISH COLUMBIA & UNIVERSITY OF SOUTHERN CALIFORNIA \\
CALIFORNIA INSTITUTE OF TECHNOLOGY & STANFORD UNIVERSITY \\
UNIVERSITY OF CALIFORNIA & UNIVERSITY OF HAWAII \\
MONTANA STATE UNIVERSITY & UNIVERSITY OF TOKYO \\
UNIVERSITY OF NEVADA, RENO & UNIVERSITY OF UTAH \\
NEW MEXICO STATE UNIVERSITY & WASHINGTON STATE UNIVERSITY \\
OREGON STATE UNIVERSITY & UNIVERSITY OF WASHINGTON
\end{tabular}

The Supporting Institutions listed above contribute to the cost of publication of this Journal, but they are not owners or publishers and have no responsibility for its content or policies.

Mathematical papers intended for publication in the Pacific Journal of Mathematics should be in typed form or offset-reproduced (not dittoed), double spaced with large margins. Please do not use built up fractions in the text of the manuscript. However, you may use them in the displayed equations. Underline Greek letters in red, German in green, and script in blue. The first paragraph must be capable of being used separately as a synopsis of the entire paper. In particular it should contain no bibliographic references. Please propose a heading for the odd numbered pages of less than 35 characters. Manuscripts, in triplicate, may be sent to any one of the editors. Please classify according to the scheme of Math. Reviews, Index to Vol. 39. Supply name and address of author to whom proofs should be sent. All other communications should be addressed to the managing editor, or Elaine Barth, University of California, Los Angeles, California 90024.

There are page-charges associated with articles appearing in the Pacific Journal of Mathematics. These charges are expected to be paid by the author's University, Government Agency or Company. If the author or authors do not have access to such Institutional support these charges are waived. Single authors will receive 50 free reprints; joint authors will receive a total of 100 free reprints. Additional copies may be obtained at cost in multiples of 50 .

The Pacific Journal of Mathematics is issued monthly as of January 1966. Regular subscription rate: $\$ 132.00$ a year (6 Vol., 12 issues). Special rate: $\$ 66.00$ a year to individual members of supporting institutions.

Subscriptions, orders for numbers issued in the last three calendar years, and changes of address should be sent to Pacific Journal of Mathematics, P.O. Box 969, Carmel Valley, CA 93924, U.S.A. Old back numbers obtainable from Kraus Periodicals Co., Route 100, Millwood, NY 10546.

The Pacific Journal of Mathematics ISSN 0030-8730 is published monthly by the Pacific Journal of Mathematics at P.O. Box 969, Carmel Valley, CA 93924. Application to mail at Second-class postage rates is pending at Carmel Valley, California, and additional mailing offices. Postmaster: Send address changes to Pacific Journal of Mathematics, P. O. Box 969, Carmel Valley, CA 93924.

PUBLISHED BY PACIFIC JOURNAL OF MATHEMATICS, A NON-PROFIT CORPORATION

Copyright $\odot 1983$ by Pacific Journal of Mathematics 


\section{Pacific Journal of Mathematics}

Vol. 107, No. $2 \quad$ February, 1983

Driss Abouabdillah, Topologies de corps $A$ linéaires $\ldots \ldots \ldots \ldots \ldots . \ldots 257$

Patrick Robert Ahern, On the behavior near a torus of functions

holomorphic in the ball $\ldots \ldots \ldots \ldots \ldots \ldots \ldots \ldots \ldots \ldots \ldots \ldots \ldots . \ldots 267$

Donald Werner Anderson, There are no phantom cohomology operations

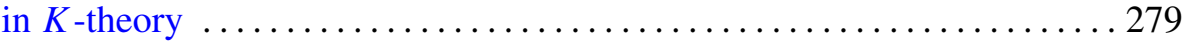

Peter Bloomfield, Nicolas P. Jewell and Eric Hayashi, Characterizations of

completely nondeterministic stochastic processes . ............. 307

Sydney Dennis Bulman-Fleming and K. McDowell, Absolutely flat

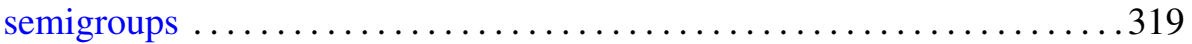

C. Debiève, On a Radon-Nikodým problem for vector-valued measures . . . 335

Dragomir Z. Djokovic, Products of positive reflections in real orthogonal

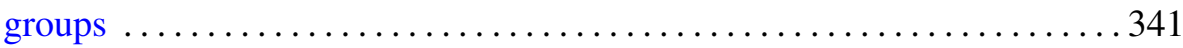

Thomas Farmer, The dual of the nilradical of the parabolic subgroups of

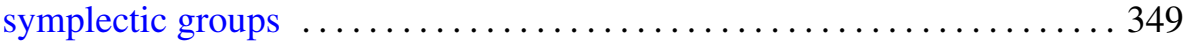

Gary R. Greenfield, Uniform distribution in subgroups of the Brauer group of an algebraic number field $\ldots \ldots \ldots \ldots \ldots \ldots \ldots \ldots \ldots \ldots \ldots . \ldots \ldots$

Paul Daniel Hill, When $\operatorname{Tor}(A, B)$ is a direct sum of cyclic groups $\ldots \ldots \ldots 383$

Hiroshi Maehara, Regular embeddings of a graph $\ldots \ldots \ldots \ldots \ldots \ldots \ldots 3$

Nikolaos S. Papageorgiou, Nonsmooth analysis on partially ordered vector spaces. I. Convex case . .............................4 403

Louis Jackson Ratliff, Jr., Powers of ideals in locally unmixed Noetherian

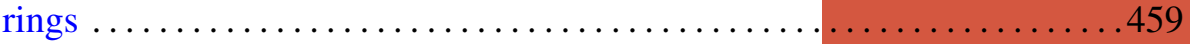

F. Dennis Sentilles and Robert Francis Wheeler, Pettis integration via the Stonian transform .......................... 